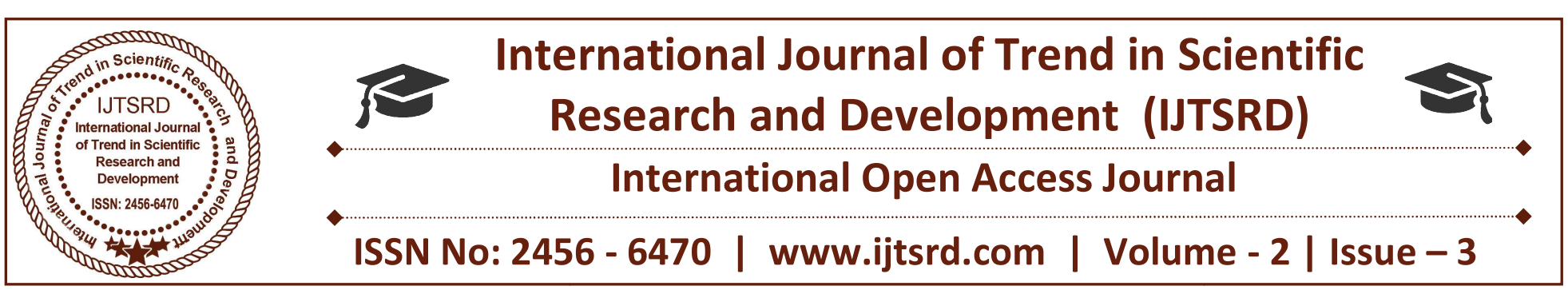

\title{
Analysis of Trust-Based Recommendation for Recommendation Model in Data Mining
}

\author{
N. Sreenivasulu \\ MCA Final Year, Department of MCA, \\ Lakireddy Balireddy College of Engineering, \\ Mylavaram, Andhra Pradesh, India
}

\author{
R. Vijaya \\ Associate Professor, Department of MCA, \\ Lakireddy Balireddy College of Engineering, \\ Mylavaram, Andhra Pradesh, India
}

\section{ABSTRACT}

As an essential strategy for Data Filtering, the recommender structures have been pulled in and made a huge amount of eagerness as far back as ten years. The past suggestion procedures and philosophies have been extensively analyzed in the information recuperation investigate gatherings, machine learning frameworks and data mining. Because of their marvelous business ask for, the suggestion structures have been adequately worked out in present day circumstances and in business zones, for instance, proposition of the thing at Amazon, proposal of music at iTunes, proposition of movies at Netflix, and whatnot. Here, we are proposing an approach called TrustSVD, which is a trust-based cross section factorization framework for thing or organization suggestions. This TrustSVD arranges various distinctive information sources into the proposal structure to diminish the data sparsity and the chilly begin starts issues and their degradation execution. An essential examination of social trust data from the few of genuine educational records tells that, the unequivocal and certain effect of the two assessments and trust must be considered for a proposition illustrate. Accordingly TrustSVD develops the best in class recommender strategy known as, SVD++ (which has usage of effect of certain and express assessed things), by additionally combining both effect of trusted and confiding in customers upon the conjecture of the things for a dynamic customer. In perspective of our canny learning of recommender systems, the proposed technique is the first to upgrade SVD++ with the social put stock in information.
Keywords: Data filtering, Trust SVD++, Recommendation, Rating

\section{Introduction}

With the rapidly creating measure of data available on the web, it changes into basic to have equipment to help customers to pick the vital a bit of online information. To fulfill this need, recommender systems have raised, e.g. there are notable recommenders for films, books, music, and so forth. For the most part in a recommender structure, we have a social event of customers and a plan of things. Every customer $\mathrm{u}$ rates a plan of articles with the guide of two or three characteristics. The recommender has the test to are expecting the rating for purchaser $\mathrm{u}$ on a nonrated thing I or to normally propose a few things for the given customer $u$ essentially in perspective of the assessments that exist starting at now. All around kind of recommender systems were inquired about: memory-based and demonstrate basically based recommenders. memory based calculations (community sifting) discover the customer thing rating lattice and make shows essentially in perspective of the evaluations of thing I by strategies for a game plan of customers whose rating profiles are most noteworthy just like that of individual $u$. show based totally frameworks separate the parameters of a model and keep only those parameters. Along these lines they might never again want to discover the rating lattice and simply keep the model parameters. Show based completely approaches are incredibly brisk after the parameters of the frame are learnt. The bottleneck for demonstrate 
based totally strategies is the coaching region, while in memory-based methodology there is no planning, however the desire (test) arrange is slower. Group arranged isolating is best when customers have imparted satisfactory scores to have essential scores with different customers; despite it performs inadequately for implied chilly start customer. Cold begin customers are new customers who've conveyed only two or three evaluations. Using resemblance based procedures; it isn't most likely going to find essentially indistinguishable customers for the reason that frosty start customers simply have a couple of assessments. Social society based recommenders; regardless, can make suggestions because of the fact that another individual is joined to an adequately generous segment of the informal organizations.

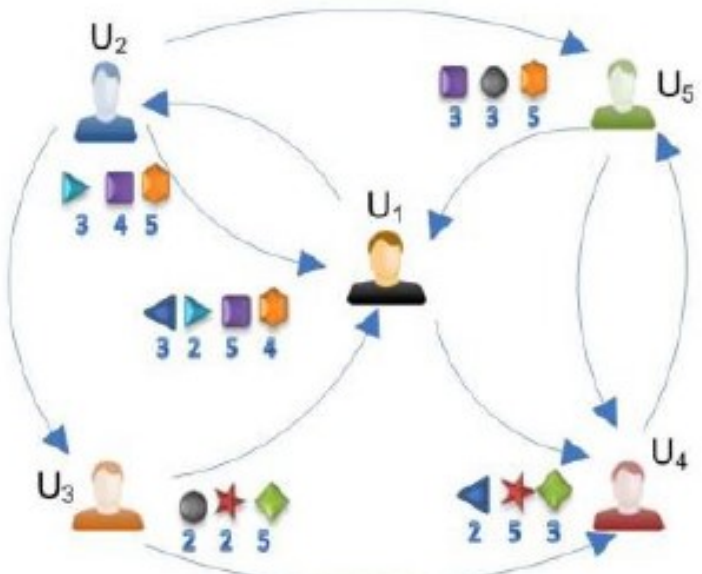

Fig.1. A Simple Social Rating Network both (unequivocal and comprehended) impact of rating and place stock in data.

\section{Related Work}

The recommender frameworks have been broadly used to offer clients with stupefying patch up proposals from monstrous aggregate most recent choices. Collaborative filtering (CF) is a champion among the most predominant ways to deal with put into influence a recommender framework. The likelihood of $\mathrm{CF}$ is that clients with equivalent decisions in the past are well while in transit to help the relative things (e.g., films, music, books, et cetera.) later on. $\mathrm{CF}$ has besides been done to obligations other than thing recommendations, in spaces which meld picture managing and bioinformatics. Regardless, CF experiences two in all cases troubles: data sparsity and cool begin. The two issues truly corrupt the ability of a recommender framework in demonstrating client proposals and along these lines the precision of reckoning a client's surveying for a dull thing. To help settle those pesters, different specialists attempt to unite social trust data into their recommendation models; given that model based absolutely CF structures beat memory in a general/sense based procedures. These methods besides regularize the client particular fragment vectors through the ponder that partners' suppositions may impact each other in proposing things. Regardless, even the tremendous execution controlled by strategies for the best in class work can be substandard stood out from that best in class diverse recommendation models which may be generally fundamentally in context of user- thing rankings. Trust-based recommender structures had been extensively contemplated, for the reason that social trust bears an elective perspective of client inclines other than thing evaluations. There are administering recommendation tries in recommender structures, particularly thing suggestion and rating want. Most algorithmic systems are perfect (or tasteful) proposed for either absolutely one of the recommendations commitments and our work impacts a recognizing quality of the rating assessment to challenge.

\section{Framework}

Around there we delineate our proposed recommendation structure called TrustSVD which is a trust based suggestion model and motivation driving framework factorization in the midst of the time spent want of client rating and thing suggestion. In social rating structures, a man can check (fuse) unmistakable 
clients as confided in companions and thus shape a party. Trust isn't symmetric; for instance, client $\mathrm{u} 1$ trusts $\mathrm{u} 3$ yet $\mathrm{u} 3$ does never again choose $\mathrm{u} 1$ as sensible. Likewise, clients can rate a game-plan of things by utilizing some of rating respects, e.g., whole numbers from 1 to 5 . Those things can be stock, movies, tune, et cetera of intrigue. The proposition issue on this work is to expect the rating that a purchaser will oblige a dim thing, for example, the cost that client $\mathrm{u} 3$ will accommodate thing i3, in context of a user- thing rating network and a userclient put stock in framework. Other all around saw recommendation issues are top- $\mathrm{N}$ thing suggestion.

Investigation on Trust: In this paper, we get a handle on the essentialness of social place stock in given as one's affirmation towards the breaking point of others in giving imperative examinations. It joins a high bore and subjective evaluation about other's capacity in offering noteworthy examinations. Trust might be what's more split into express trust and obvious trust. Express trust recommended trust declarations coordinate controlled by the clients. For instance, clients in Epinions and Ciao can consolidate specific clients into their place stock in records. Through assessment, grasped trust is the association that isn't plainly delegated by clients and this is as every now and again as possible gathered by procedure for various information, containing client rankings. In this article, we just mishandle the estimation of express trust for rating figure. We organize the trust-alike relationship as the social affiliations which are close with, yet weaker (or more unmistakable uproarious) than social recognize. The similarities are that the two mixes of affiliations propose client diverse decisions to a particular degree and in like way gainful for recommender frameworks, in the interim as the capabilities are that trust-alike affiliations are as frequently as possible weaker in power and possible to be additional rambunctious. Standard cases are affiliation and enrollment for recommender systems. Disregarding the way that those relationship in like way suggest that clients may in addition have a gainful relationship with particular likeness, there might be no affirmation that such a fine assessment ordinarily exists and that the affiliation will be enthusiastic. It's far magnificently seen that organization can be made generally in context of separated individuals from the family, near to accomplices and partners, which does never again continually rate in every practical sense vague decisions. Concur with is a mind boggling thought with some of homes, including asymmetry and space reliance, which trust-alike affiliations won't not keep up, e.g., family relationship is undirected and territory sensible. For clearness, in this flyer we infer concur with clients or trust neighbors to as the association set of clients who concur with an energetic purchaser (i.e., trusters) and of clients who're depended upon by strategies for the dynamic client (i.e., trustees).

Framework Factorization Some of the best recognize of torpid factor models are develop totally regarding lattice factorization. In its basic casing, arrange factorization depicts the two things and customers through vectors of segments assembled from thing rating styles. High correspondence among thing and customer factors realizes a proposition. Those frameworks have created as well known recently by strategy for joining honest to goodness flexibility with farsighted precision. So likewise, they offer high flexibility for showing different certifiable nearness conditions. One vitality of lattice factorization is that it stipends joining of extra convictions. Right when unequivocal feedback isn't available, recommender structures can induce singular choices the usage of comprehended information, which indirect shows supposition by methods for looking direct, for instance, buy records, scrutinizing history, search for outlines, or even mouse moves. Certain comments commonly show the proximity or nonappearance of an event, so it is overall addressed by strategy for a thickly stuffed system. System factorization plans layout customers and things to a joint sit point of view locale of dimensionality $f$, to such a degree, to the point that buyer challenge associations are shown as internal things around there. As requirements are, everything I is related to a vector $\mathrm{q}$, and every customer $\mathrm{u}$ is connected with a vector $\mathrm{p}$. For a given customer $\mathrm{u}$, the segments of pu measure the measure of interest the buyer has in things that are high on the relating parts, once more, high gauge or dreadful. The accompanying spot thing, gets the exchange between purchaser $\mathrm{u}$ and question $\mathrm{I}-$ the customer's typical excitement for the thing's characteristics. This approximates singular u's assessing of question $I$ that is shown by methods for $r$ ui, basic to the measure. The basic errand is figuring the mapping of each inquiry and individual to perspective vectors $q, p$ has a place with $f$. After the recommender machine completes this mapping, it can without inconvenience assess the rating a man will provide for any dissent by techniques for using Equation 1. Such a model is enthusiastically related to 
solitary esteem deterioration (SVD), a properly mounted approach for perceiving lethargic semantic factors in data recuperation. Applying SVD in the group arranged isolating zone requires considering the individual inquiry score organize. This routinely fabricates issues in view of the nonsensical piece of lacking regards because of deficiency in the individual thing assessments organize. Standard SVD is indistinguishable while dominance around the system is divided. Plus, rashly watching out for handiest the respectably few perceived segments is strikingly vulnerable to over fitting.

TrustSVD Our TrustSVD exhibit is produced on top front line show implied as SVD++ proposed by Koren [12]. The point of view behind SVD++ is to think about customer/thing inclinations and influence state of-the workmanship assessed things close to client/thing particular vectors on rating desire. Formally, the rating for customer $\mathrm{u}$ on thing $\mathrm{j}$ is ascertained by using:

$$
\hat{r}_{u, j}=b_{u}+b_{j}+\mu+q_{j}^{\top}\left(p_{u}+\left|I_{u}\right|^{-\frac{1}{2}} \sum_{i \in I_{u}} y_{i}\right),
$$

Where $\mathrm{b} \mathrm{u}, \mathrm{bj}$ address the rating inclination of customer $u$ and thing $\mathrm{j}$, independently; $\mathrm{m}$ is the overall typical rating; and yi implies the specific effect of things assessed by customer $\mathrm{u}$ in the past on the evaluations of cloud things later on. Subsequently, we can enhance the trust ignorant SVD++ show/by joining both the unequivocal and comprehended effect of trust.

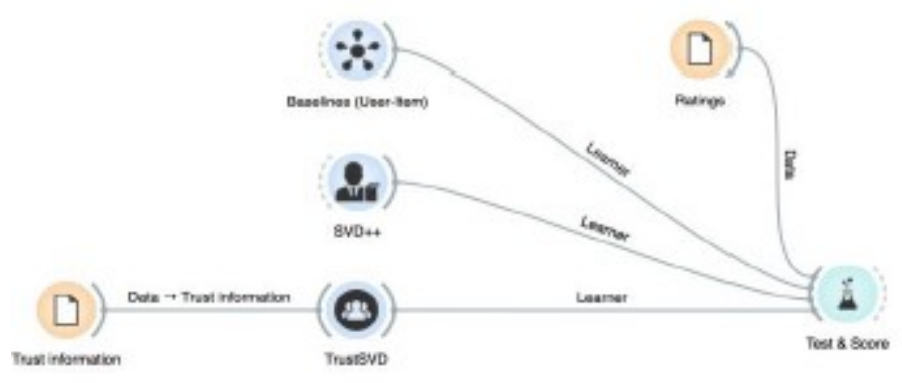

Fig.2. A Basic trust SVD Model.

Model Learning: In Model adapting, to begin with, we heedlessly present the disintegrated vectors and systems with little regards. By then, we keep setting up the model until the point that the mishap work is consolidated. Specifically, we process variable points, and a short time later revive factors by the slant fall system. Finally, we reestablish the informed vectors and systems as yield.

\section{Experimental Results}

To make our novel suggestion exhibits with customer trust and thing rating we drove our exploratory on windows organize by realizing our approach in with the help of java programming vernacular. We have inserted our suggestion appear in light of epinions dataset with evaluations data and place stock in data. We perform rating gauge in light of certain and express evaluating appraisal. Trial comes to fruition exhibit that our Trust based Recommendation appear with thing evaluations can better perform over existing trust based models and rating based models.

Here is our verification of performance.

i. Implicit rating prediction value

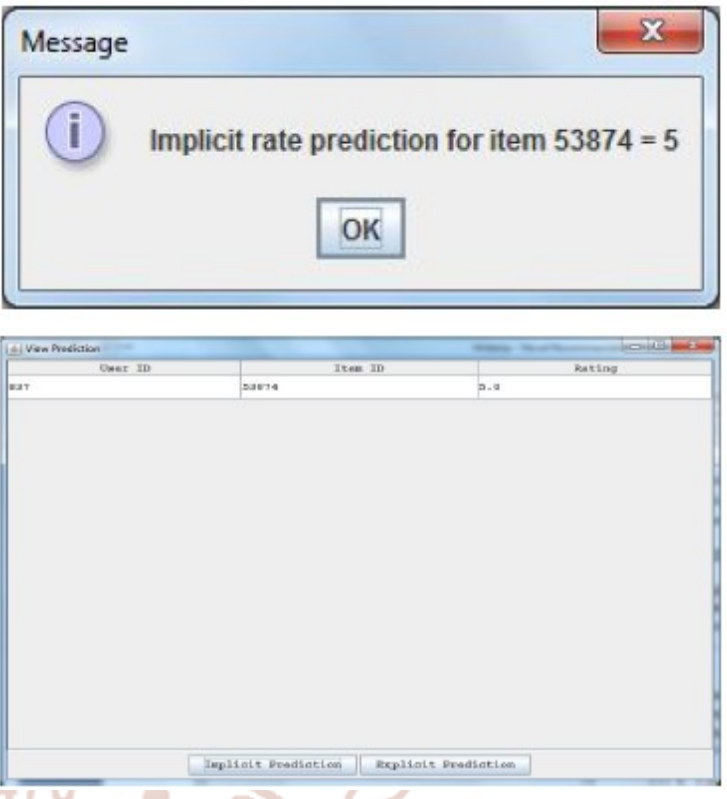

ii. Explicit rating prediction value.
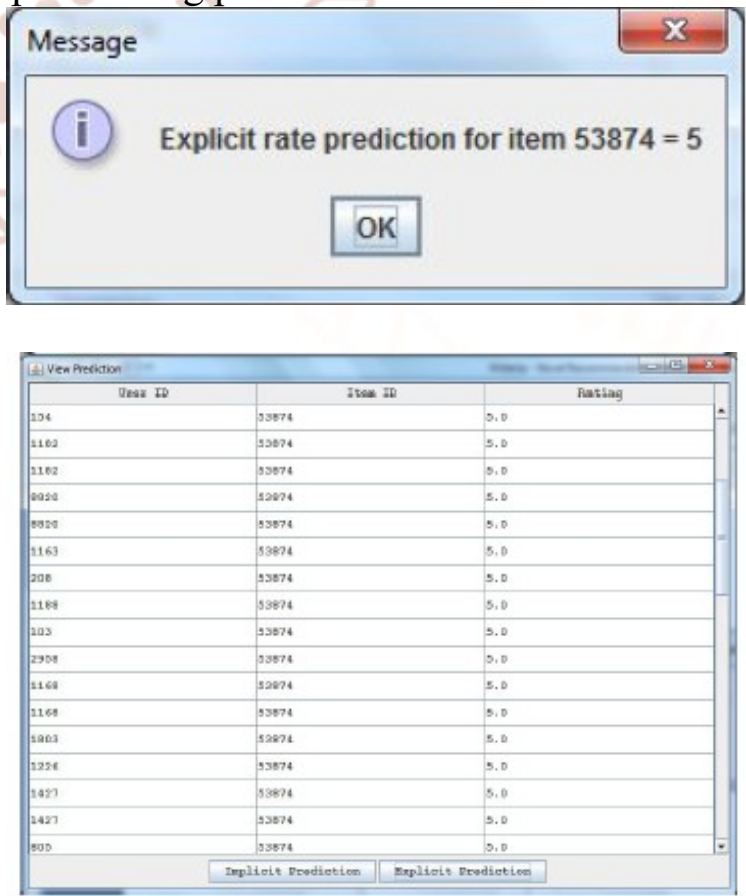


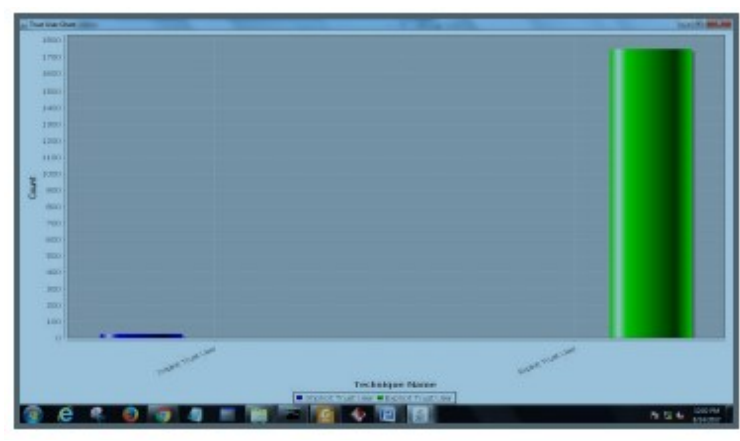

\section{Conclusion}

Recommender structures made broad progression in the midst of the latest decade while a couple of substance based, shared, and crossover systems were proposed and different "business-quality" structures have been produced. In setting of this paper we exhibit an absolutely stand-out trust based system factorization indicate which is made with trust information and dissent rating. Our novel approach, TrustSVD, thinks about both the unequivocal and certain effect of assessments and of put stock in data while anticipating examinations of darken articles. Both the trust effect of trustees and trusters of dynamic clients are worried in our model. Further, a weighted regularization technique is adjusted and connected with moreover regularize the advancement of client and thing cautious sit still trademark vectors. Computational diserse nature of TrustSVD displayed its accommodation of scaling up to sweeping scale information units. Finish trial happens on the four bona fide far reaching information units affirmed that our approach TrustSVD vanquished both trust-and examinations based techniques (ten models in all around) in insightful accuracy crosswise over completed extraordinary testing points of view and each and every through client with unmistakable place stock in stages. We expected that our approach can particularly direct the data sparsity and cold start begins disturbs of recommender frameworks.

\section{References}

1) B. Yang, Y. Lei, D. Liu, J. Liu, "Social collaborative filtering by trust," in Proc. 23rd Int. Joint Conf. Artif. Intell., 2013, 2747-2753.

2) H. Fang, Y. Bao, J. Zhang, "Leveraging decomposed trust probabilistic matrix factorization for effective recommendation," in Proc. 28th AAAI Conf. Artif. Intell., 2014, pp. 30-36.

3) Y. Koren, R. Bell, C. Volinsky, "Matrix factorization techniques for recommender systems," Computer, vol. 4, no. 8, pp. 30- 37, Aug. 2009.

4) Y. Koren, "Factor the neighbors: Scalable, accurate collaborative filtering," ACM Trans. Know. Discovery Data, vol. 4, no. 1, pp. 1:1-1:24, 2010.

5) Y. Koren, "Factorization meets the neighborhood: A multifaceted collaborative filtering model," in Proc. 14th ACM SIGKDD Conf. Know. Discovery Data Mining, 2008, pp. 426-434.

6) G. Guo, J. Zhang, N. Yorke-Smith, "TrustSVD: Collaborative filtering with both explicit and implicit influence of user trust and of item ratings," in Proc. 29th AAAI Conf. Artif. Intell., 2015, pp. 123- 129.

7) G. Adomavicius, A. Tuzhilin, "Toward the next generation of recommender systems: A survey of the state-of-the-art and possible extensions," IEEE Trans. Know. Data Eng., vol. 17, no. 6, 734-749, Jun. 2005.

8) Y. Huang, X. Chen, J. Zhang, D. Zeng, D. Zhang, $X$. Ding, "Single-trial ERPs denoising via collaborative filtering ERPs images," Neurocomputing, vol. 149, pp. 914-923, 2015.

9) X. Luo, Z. Ming, Z. You, S. Li, Y. Xia, and H. Leung, "Improving network topology-based protein interactome mapping collaborative filtering," Knowl.-Based Syst., vol. 90, pp. 23-32, 2015.

10) A. Sahoo, K. Kant, P. Mohapatra, "Improving BGP convergence delay for large-scale failures," in Proc. Dependable Syst Net, 2006, pp. 323-332.

11) B. Bassiri, S. S. Heydari, "Network survivability large-scale regional failure scenarios," in Proc. 2nd Canadian Conf. Comput. Sci. Softw. Eng., 2009, pp. 83-87.

12) T. Bu, N. Duffield, F. L. Presti, D. Towsley, "Network tomography on general topologies," SIGMETRICS Perform. Eval. Re., vol. 30, pp. 21-30, Jun. 2002.

13) G. Guo, J. Zhang, D. Thalmann, "A simple but effective method to incorporate trusted neighbors in recommender systems," Proc. 20th Int. Conf. User Model., Adaptation Personalization, 2012, pp. 114- 125.

14) H. Ma, H. Yang, M. Lyu, I. King, "SoRec: Social recommendation using probabilistic matrix 
factorization," Proc. 31st Int. ACM SIGIR Conf. Res. Develop. Inform. Retrieval, 2008, pp. 931940.

15) H. Ma, D. Zhou, C. Liu, M. Lyu, I. King, "Recommender systems with social regularization," Proc. 4th ACM Int. Conf. Web Search Data Mining, 2011, pp. 287-296
16) M. Jamali, M. Ester, "A matrix factorization technique with trust propagation for recommendation in social networks," Proc. 4th ACM Conf. Recommender Syst., 2010, pp. 135142.

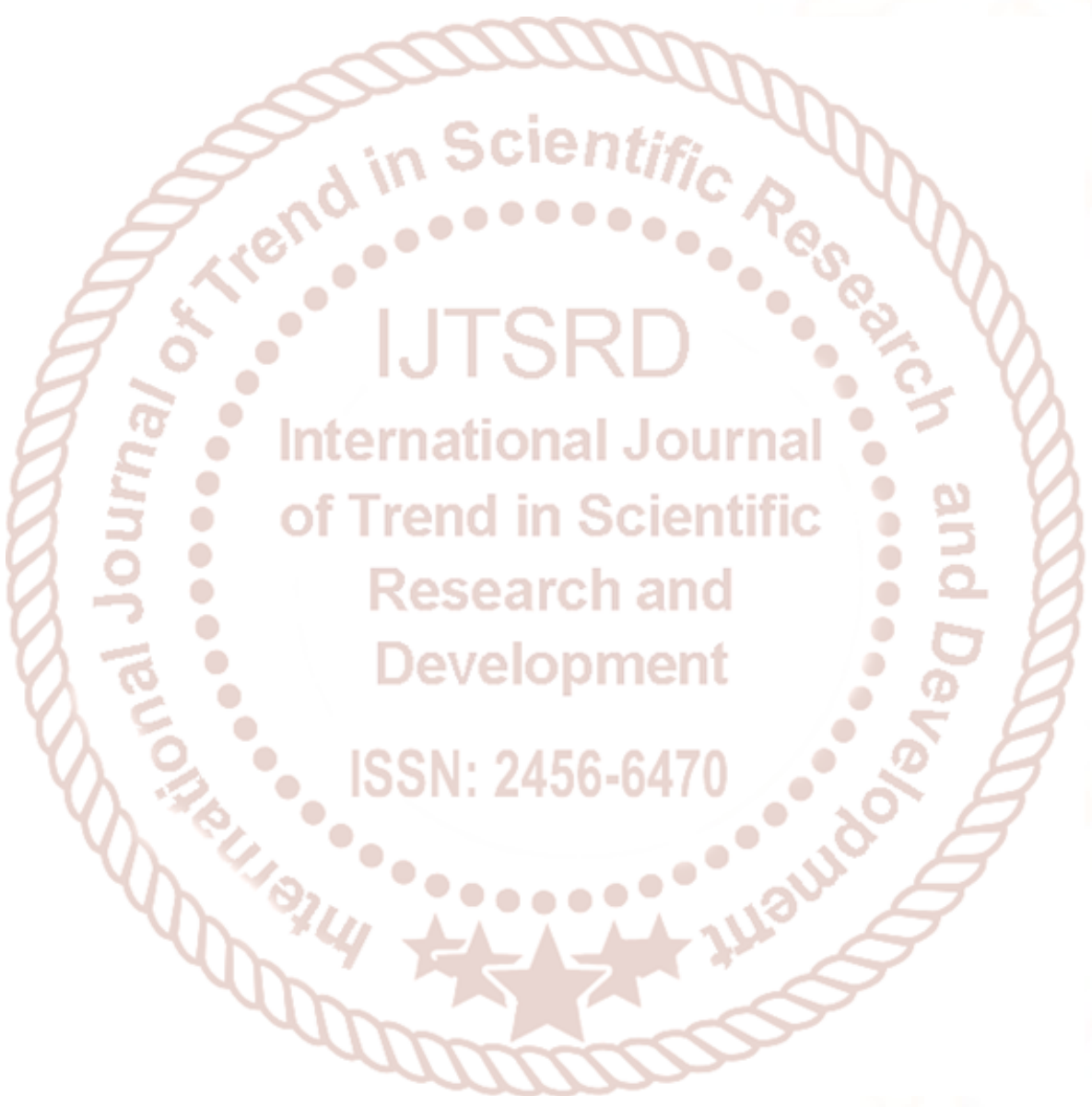

